\title{
REAL TIME ESTIMATION OF THE HEAVING AND PITCHING MOTIONS OF A SHIP USING A KATMAN FILTER
}

by

MICHAEL TRIANTAFYIIOU

MICHAEL ATHANS

IABORATORY FOR INFORMATION AND DECISION SYSTEMS MASSACHUSETTS INSTITUTE OF TECHNOLOGY

CAMBRIDGE, MASSACHUSETTS 02139

* Reprinted from Proc. OCEANS' 81, Boston Mass., September 1981. Research Supported by NASA Ames and Langley Research Center under grant NGL-22-009-124. 
REAL TIME ESTIMATION OF THE HEAVING AND PITCRING MOTIONS OF A SHIP, USING A RAIMAN FILTER

Michael Triantafyllou Michael Athans

Ocean Engineering Dept., Electrical Engineering and

and Iaboratory Computer Science Dept... Massachusftts Institute of Technology

\section{ABSTRACT}

Real time ship motion estimation is of interest to naval ship operators for vertical aircraft landing, as well as to the offshore industry for installing rigs and transferring equipment in rough seas.

In the present study the estimation of the heave and pitch motion of a ship is considered, using Kalman filtering techniques. A significant part of the study is devoted to constructing appropriate models for the sea and the ship.

The governing equations are obtained from hydrodynamic considerations in the form of linear differential equations with frequency dependent coefficients. In addition, non-minimum phase characteristics are obtained due to the spatial integration of the water wave forces.

The resulting transfer matrix function is irrational and non-minimum phase. The con ditions for a finite-dimensional aporoximation are considered and the imoact of the various parameters is assessed. A numerical application is considered for a DD-963 destroyer.

\section{INTRODUCTION}

The real time estimation of the ship motions, velocities and accelerations $1 \mathrm{~s}$ very important for applications in rough seas, such as the landing of aircraft or helicopters on ships, the transfer of cargo, or heavy equipment at sea and offshore rig installation.

In a study on the vertical landing if aic craft on destroyers, it was found that the modeling of the ship motions plays a very important role in any automatic landing control scheme $[4]$.

In the present study the accurate finite dimensional modeling of the heave and pitch motions of a ship is considered. for application in the real time estimations of the motions, velocities and accelerations using Kalman filter techniques. Only two measurements are assumed available, the heave and pitch motion.

\section{EQUATIONS OF MOTION}

\section{2a. Definitions}

The rigid body motions of a ship in six degrees of freedom are shown in Figure 1: We define the $x_{1} z_{1}$ plane to coincide with the symmetry plane of the ship, with the $z_{1}$ axis pointing vertically upwards, and the $y_{1}$ axis to port, so as to obtain an orthogonal right-hand system. Then the linear motions along the $x_{1}, y_{1}, z_{1}$ axes are surge, sway and heave respectively. We consider small motions so that the tensor of angular displacements can be replaced by a vector of small angular displacements, which are roll, pitch, yaw around the $x_{1}, y_{1}, z_{1}$ axes respectively. The assumption of small wave induced motions is justified, given the large mass of the vessel and the small wave amplitude to length ratio. Very good predictions have been obtained in the literature using this assumption $[5]$.

\section{2b. Dexivation of the Equations of Motion}

The complex problem of wave induced motions can be simplified within linear theory as follows: The incident waves are diffracted by the vessel, while waves are radiated away from the ship as she oscillates. The diffraction waves can be found by assuming the vessel motionless within the incident waves, while the radiation waves are found by oscillating the vessel in calm water [5]. The force caused by the incident ard diffraction waves is called the exciting force and can be separated in the part caused by the incident waves (Froude-Krylov force) and the diffraction force. The force caused by the radiation waves is proportional to the motion amplitude so if the motion is

$$
\underline{x}(t)=\underline{x}_{0} e^{i \omega_{0} t}
$$


with xo complex, then the radiation force is

$$
\underline{F}_{x}(t)=T\left(\omega_{0}\right) \underline{x}_{0} e^{1 \omega_{0} t}
$$

The frequency dependent constant $T\left(w_{0}\right)$ can be proved to include the hydrostatic terms, terms modeling the energy loss due to the outgoing waves (damping terms). and terms representing the inertia of the entrained fluid (added mass terms) [1]. For this reason we can rewrite (2) as

$$
F_{X}(t)=-A\left(w_{0}\right) \ddot{x}(t)-B\left(w_{0}\right) \dot{x}(t)-C x(t)
$$

where the added mass $A(\omega)$ and damping $B(\cdot \omega)$ are frequency dependent, because the radiation waves are different at various frequencies.

The exciting force can be written as

$$
F_{r}(t)=F_{0}\left(\omega_{0}\right) e^{i \omega_{0} t}
$$

so by Newton's law

$$
-H x_{0} \omega^{2} 0 e^{i \omega_{0} t}=\left[F_{0}\left(\omega_{0}\right)+\left[A\left(\omega_{0}\right) \omega^{2} 0-\right.\right.
$$$$
\left.\left.i \omega_{0} B\left(\omega_{0}\right)-c\right] x_{0}\right\} e^{i \omega_{0} t}
$$

By aropping $e^{i \omega_{0} t}$, we can rewrite equation (4) as:

$\left\{-\left[\psi+\dot{A}\left(\omega_{0}\right)\right] \omega_{0}^{2}+i \omega_{0} B\left(\omega_{0}\right)+C\right\} x_{0}=F_{0}\left(\omega_{0}\right)$

vitimately, we wish to obtain the response in a random sea, so equation(4a) must be extended for a random sea. This can be done by obtaining the inverse Fourier transfrom of (4), i.e.

$$
\begin{aligned}
& \int_{-\infty}^{\infty} R_{\alpha}(t-\tau){ }_{\infty}^{n}(\tau) d \tau+\int_{-\infty}^{\infty} k_{v}(t-\tau): x(\tau) d \tau+ \\
& +c x(t)=\sum_{-\infty}^{i} K_{f}(t-\tau) n(t) d \tau
\end{aligned}
$$

where $K_{\alpha}, K_{v}, K_{f}$ the inverse Fourier transform of $-\omega^{2}[M+A(\omega)], i \omega B(\omega)$ and $F_{0}(\omega)$ respectively. The random undisturbed wave elevation is denoted by $n(t)$. Equation (5) is not popular with hydrodynamicists because the effort required to evaluate the kernels $K_{\alpha}, K_{v}, K_{f}$ is by far greater than to find the adaed mass, damping and exciting force. For this reason, equation (5) is rewritten in a hybrid form as follows:

$$
\begin{aligned}
-[M+A(\omega)] & \ddot{x}(t)+B(\omega) \dot{x}(t)+C \times(t) \\
& =F(\omega) \cap(t) .
\end{aligned}
$$

This is an integro-differential equation for differential equation with frequency dependent coefficients), whose meaning is in the sense of equation (5). It should be noted that $-\omega^{2} A(\omega), i \omega B(\omega)$ are caused by the same radiation waves. so they are related by the Cramers-kröning relations of causal systems.

\section{2c. Speed Effects}

When the ship is heaving with a small angle $\theta$ and at the same time is moving forward with speed $U$, then a heave velocity results, which is $\&=v \theta$. The effect of the forward speed, therefore, is to couple the various motions by speed dependent coefficients. As it can be found in Appendix 1, there are simplified expressions for the added mass, damping and exciting force with a parametric dependence on the speed $v$.

\section{2d. Frequency of Encounter}

An aditional effect of the ship speed is the change in the frequency of encounter. If the incident wave has a frequency $\omega$ then the frequency of encounter $\omega_{e}$ is. for deep water:

$$
\omega_{e}=\omega \frac{\omega^{2}}{g} v \cos \phi
$$

Although the exciting force changes with frequency $\omega$, its value is the same as if the frequency were $\omega$ (plus any speed dependent terms).

2e. Equations of Motion

within linear theory and using the ship symmetry, the heave and pitch motions are not coupled with the group of sway, roll. yaw motions. We can express the heavepitch motions therefore as:

$$
\begin{gathered}
\left\{\left[\begin{array}{ll}
m & 0 \\
0 & I_{y}
\end{array}\right]+\left[\begin{array}{lll}
A_{3} & A_{3} \\
A_{5} & A_{5}
\end{array}\right]\right\} \underline{x}_{v}+\left[\begin{array}{lll}
B_{3} & B_{3} & 5 \\
B_{5} 3 & B_{5} 5
\end{array}\right] \underline{x}_{v}+ \\
+\left[\begin{array}{ll}
C_{3} & C_{3} 5 \\
C_{53} & C_{55}
\end{array}\right] \underline{x}_{v}=\left[\begin{array}{l}
F_{3} \\
F_{5}
\end{array}\right] \eta
\end{gathered}
$$

Where Aij,Bij,Cij tise added mass, damping, hydrostatic coeficient matrices respectively; $F$, the $u x c i t i n g$ forces; $n$ the wave elevation;

$$
\underline{x}_{v}=\left\{x_{3}, x_{5}\right\}^{T}
$$

The subscriot, refers to heave and = to pitch (consistent with the hvdrodynamic iterature). The frequency and velocity dependence is not written explicitly,but is understood, as described in the previous sections.

\section{2f. Exciting Force Approximation}

The heave exciting force amplitude presents several peaks and zeros as can be seen in figure 2. W1 chin the wave frequency range, on $1 y$ the first peak is important, so that a relatively simple inite dimensional approximation can be used.

As it was mentioned before, the exciting force depends on the wave frequency $w$ and 
varies with frequency $\omega$, while it is parametrically dependent on the speed and the wave angle.

In order to model the DD-963 destroyer, the M.I.T. five degrees of freedom seakeeping program [ 2$]$ was used to derive hydrodynamic results. The following model was derived to model the shape of the heave force at $V=0$ and $\phi=0$ (no speed, head seas)

$$
F_{3}(s)=\frac{\alpha_{1} n}{\left[1+2 J \frac{8}{\omega_{a}}+\frac{s^{2}}{\omega^{2}}\right]^{2}}
$$

Where $J=0.707$ chosen for optimal response of a second order system, $\alpha_{1}$ a constant determined from hydrodynamic data, $n$ the wave elevation and $w_{a}$ the corner frequency.

This approximation was extended to include the effects of speed and wave angle by defining:

$$
\omega_{a}=\sqrt{\frac{2 \pi g}{L \cos \phi+B}}+\frac{2 \pi}{L \cos \phi+B} 0 \cos \phi
$$

where $I$ is the ship length, B the beam.

For long waves, the heave force and the pitching movement are $90^{\circ}$ out of phase. This means that the transfer function between heave and pitch is a non-minimum phase one, because the amplitude is constant, while the phase is $90^{\circ}$. We choose to attribute the non-minimum phase to pitch. Also, the pitch angle tends to the wave slope for large wavelengths,80 the pitching moment can be written as

$F_{5}=\alpha_{2} \frac{1-s / \omega_{0}}{1+s / \omega_{0}}$

$$
\frac{s^{2} \cos \phi}{\left[1+2 J \frac{s}{\omega_{a}}+\left(\frac{s}{w_{a}}\right)^{2}\right]^{2}} n
$$

Where $\alpha_{2}$ a constant to be determined, $\omega$ is the same (for simplicity) as in equa-a tion (11), while $w_{0}$ models the non-minimum phase characteristics, and was chosen to be equal to the wave spectrum modal frequency.

\section{Added Mass and Damping}

By using equation (9), we can rewrite the equations of motion as

$$
\left.\begin{array}{c}
{\left[T_{33} s^{2}+M s^{2}+C_{33} T_{35}+U s T_{3}+C_{35}\right.} \\
T_{53} s^{2}-U s T_{33}+C_{3} I_{s^{2}}+T_{55} s^{2}-U_{T_{33}}+C_{55}
\end{array}\right]
$$

Here we construct a simplified model where

$$
\text { Tif = Aif }-\frac{\text { Bij }}{i \omega}
$$

with Aij,Bij to be evaluated from the hydrodynamic data.

\section{WAVE SPECTRUM MODELING}

The waves in a specific location are composed of waves generated by the local wind and waves generated by a distant storm, which are usually characterized as swell. As a result, the wave spectrum contains two peaks, one very narrow at low frequencies (swell), and one relatively wide and at higher frequencies (local storm).

The Bretschneider spectrum was chosen for the present application, which allows one to specify both the intensity (significant wave height $H$ ) and the location of the peak (modal frequency $w$ m). Two such spectra can be used to model a double peaked spectrum. The form of the spectrum has as follow:

$$
s(\omega)=\frac{1.25}{4} \mathrm{H}^{2} \frac{\omega_{n}^{4}}{\omega^{5}} \exp \left\{-1.25\left(\frac{\omega_{m}}{\omega}\right)\right\}
$$

The spectrum was developed by Bretschneider for the North Atlantic, for unidirectional seas, with unlimited fetch, infinite depth and no swell. It was developed to satisfy asymptotic theoretical predictions and to fit North Atlantic data. It was found to fit reasonably well in any sea location. The spectrum, for ship coordinates, has as follows

$$
s\left(\omega_{e}\right)=\left[\frac{s(\omega)}{d \omega_{e} / d \omega}\right] ; \omega=f\left(\omega_{e}\right)
$$

where

$$
\omega=f\left(\dot{\omega}_{e}\right)=\frac{-1+\sqrt{1+4 \omega_{e} \frac{0 \cos \phi}{g}}}{2 \frac{0}{g} \cos \phi}
$$

A rational approximation was found to (15) subject to (17) in the following form

$$
s_{a}\left(\omega_{e}\right)=\frac{1.25}{4 \omega_{m}} B^{2} B(\alpha)
$$

where $B(\alpha), \omega_{0}(\alpha)$, functions

$$
\left.\frac{\omega_{e}^{4}}{\left[1+\left(\frac{\omega_{e}}{w_{0}(\alpha)}\right)^{4}\right.}\right]^{3}
$$

of $\alpha=\frac{\nabla}{g} \omega_{m} \cos \phi$

The corresponding causal system has transfer function:

$$
H_{a}(s)=\sqrt{S_{0}} \frac{s^{2}}{\left[1+2 J \frac{s}{\omega_{0}}+\left(\frac{s}{\omega_{0}}\right)^{2}\right]}
$$

where

$$
s_{0}=\frac{1.25}{4 \omega_{m}} \mathrm{H}^{2} \text { B }(\alpha) \quad J=0.707
$$

(21ab) 
It should be noted that the Bretschneider sectrum $S_{f}(w)$ is related to the actual spectrum $s(\omega)$, defined as the fourier transform of the autocorrelation, by the relation

$$
s_{b}(\omega)=\frac{1}{\pi} s(\omega) . \quad \omega>0
$$

\section{STATE SPACE MODEI}

If refers to the equations modeling the sea, fo the equations for the force and m to the ship equations, then the overall equations are written as

$$
\begin{aligned}
& \underline{\dot{x}}=\mathrm{A} \underline{\underline{x}}+\mathrm{BW} \\
& y=C \underline{\underline{y}}
\end{aligned}
$$

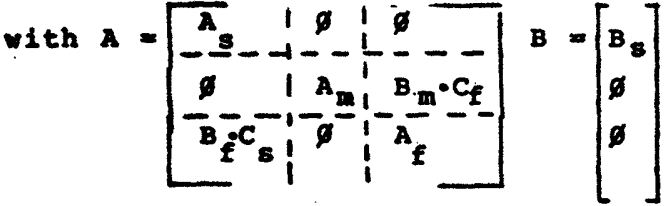

(24abcd)

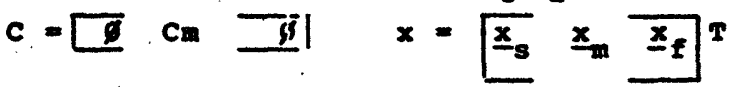
and $A_{8}=\left[\begin{array}{cccccc}0 & 1 & 0 & 0 & 0 & 0 \\ -\omega_{0}^{2} & -2 J \omega_{3} & 0 & \omega_{0}^{2} & 0 & 0 \\ 0 & 0 & 0 & 1 & 0 & 0 \\ 0 & 0 & -\omega_{0}^{2} & -2 J \omega_{0} & 0 & 0 \\ 0 & 0 & 0 & 0 & 0 & 1 \\ 0 & 0 & 0 & 0 & -\omega_{0}^{2} & -2 J \omega_{0}\end{array}\right]$ $=\left[\begin{array}{cccc}0 & 1 & 0 & 0 \\ B_{1} & B_{2} & B_{3} & B_{4} \\ 0 & 0 & 0 & 1 \\ B_{3} & B_{6} & B_{7} & B_{3}\end{array}\right]$ $A_{f}=\left[\begin{array}{lllll}0 & 1 & 0 & 0 & 0 \\ -w^{2} & -2 J w & w^{2} & 0 & 0 \\ 0 & 0 & 0 & 1 & 0 \\ 0 & 0 & -w^{2} & -2 J \omega_{a} & 0 \\ \theta_{2} & \theta_{2} & \theta_{3} & \theta_{4} & 1\end{array}\right]$

(25abc)

$$
B_{8}=\left[\begin{array}{llllll}
0 & 0 & 0 & 0 & 0 & 1]^{T}
\end{array}\right.
$$

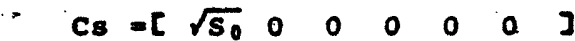$$
B_{f}=\left[\begin{array}{lllll}
0 & 0 & 0 & 1 & 0
\end{array}\right]^{\top}
$$$$
C_{f}=\left[\begin{array}{l:l:l:l}
\alpha_{1} \cdot \omega^{2} & 0 & 0 & 0 \\
0 & 0 & 0 & 0 \\
0 & \alpha^{2} \cos \phi
\end{array}\right]
$$$$
c_{f}=\left[\begin{array}{llll}
1 & 0 & 0 & 0 \\
0 & 0 & 1 & 0
\end{array}\right]
$$

where

$$
\text { \{Dif }\}=\{A i j\}^{-1}
$$

$$
\left.\left[\begin{array}{llll}
B_{1} & B_{2} & B_{3} & B_{4} \\
B_{5} & B_{6} & B_{7} & B_{4}
\end{array}\right]=f_{15}\right\}\left[\begin{array}{llll}
C_{33} & B_{33} & C_{35} & B_{35} \\
C_{53} & B_{53} & C_{55} & B_{55}
\end{array}\right]
$$

$$
\text { and } \begin{aligned}
\theta_{1} & =-\left(2 J \omega_{a}^{5}+\omega_{a}^{4}\right) \\
\theta_{2} & =-\left(\omega_{a}^{4}\left(4 J^{2}-1\right)+2 J \omega_{a}^{3}\right) \\
\theta_{3} & =\omega_{a}^{4}+2 J \omega_{a}^{5} \\
\theta_{4} & =-\omega_{a}^{4}
\end{aligned}
$$

(30abcd)

\section{KATMAN FILTER}

The gyroscopes of the ship can provide very accurate measurements of angles. The linear motions require the installation of accelerometers. For simplicity, in the present application it is assumed that the heave and pitch motions are measured only.

The accuracy of the measurements can be very good. The noise is due primarily to the structural vibrations of the ship, which for the present application were found to be as much as $0.5 \mathrm{ft}$. in molitude, and with frequency content close to $12 \mathrm{rad} . / \mathrm{sec}$. (as opposed to the wave frequencies, which are 1 ess than $1 \mathrm{rad} . / \mathrm{sec}$.

\section{APPLICATION:}

An example has been worked out for a DD-963 destroyer, whose length $I$, bean $B$ and draft $T$ are

$$
I=529 \mathrm{ft} ., B=55 \mathrm{ft} ., \mathrm{T}=18 \mathrm{ft} \text {. }
$$

The hydrodynamic characteristics were obtained using the M.I.T. sea-keeping program $[2]$, and were approximated as outlined above, for speed $\mathrm{U}=21$ ft./sec. and wave angle $0^{\circ}$

Subsequently, a Ralman filter was designed, using the M.I.T.-LIDS software [3] and for driving noise and measurement noise intensities:

$$
v_{1}=\pi \quad \sigma_{2}=\left[\begin{array}{lr}
0.75 & 0 \\
0 & 0.0003
\end{array}\right]
$$

corresponding to sea state 5 (H $=10 \mathrm{ft} ., \mathrm{um}=0.72$ rad./sec.

The filter poles are within a radius of $1.3 \mathrm{rad} . /$ sec. A typical simulation of heave and pitch motions and their predictions is given in figures 3,4 (assuming exact knowledge of the sea paraneters $H$ and $\left.\omega_{p}\right)$. By using the same filter for various combinations of $H, w$ (other than the design values) it was found that the performance of the filter depends primarily or the accurate estimation of the modal frequency $\omega_{m}:$ Figures 5 and 6 .

The simulations indicate the good accuracy that can be obtained by using the present formulation. The most important parameter is the mnial frequency, whose influence, although omooth, is quite critical. The at sea estimation of the modal frequency can be done by using the zero up-crossing 
period $\bar{x}$. Typically

$$
u_{m}=0.7 \frac{2 \pi}{T}
$$

A scheme for on-line estimation of $w_{\text {m }}$ is currently tested by extending the Xalman filter to estimate wm. Finally figure 7 illustrates the influence of a second peak (swell) on the performance of the filter. It is clearly seen that the second peak must be modelled, if accurate predictions are desired.

\section{SUMALARY}

A finite dimensional approximation of the heave and pitch equations of motion of a ship, as obtained from hydrodynamic theory, has been outlined. The important features are:

(a) the frequency dependence of the coefficients of the equation of motion

(b) the non-minimum phase characteristics between heave and pitch, due to the spatial integration of the hydrodynamic forces

(c) the dependence of the hydrodynamic quantities on the speed of the vessel and the wave angle.

A Kalman filter can be used to estimate the motions, velocities, etc., whose performance primarily depends on an accurate estimation of the sea spectrum modal frequency. Very good estimation is obtained when all the features, mentioned above, are included.

\section{ACKNOWLEDGEYGENS}

The research presented in this paper was supported by a grant from NASA-Ames Research Center under Grant NGL-22-009-124, and was conducted at the M.I.T. Laboratory for Information and Decision Systems.

\section{REFEREAVEES}

(1) Salvesen, N.,Tuck ,Faltinsen,0.,"Ship Motions and Ship Loads," Trans. SNAME, 1970.

(2) "5-Degrees of Freedom Seakeeping Program Manual," Design Laboratory, Ocean Engineering Dept., M.I.T., 1974, Cambridge, Mass.

(3) "Dynamic Control systems Software --User's Manual," Laboratory for Information and Decision Systems, M.I.T., 2980, Cambridge, Mass.

(4) MeMuldroch, C.G., "Virot Controls for Shipboard Landing," Laboratory fox Information and Decision Systems, Report IIDS-TH-928, 1979, M.I.T. ,Cambridge, Mass.

(5) Newman; J.N., "Marine Hydrodynamics," M.I.T. Press, 1978, Cambridge, Mass.
APPEIDIX 1

The following relations provide the hydrodynamic coefficients and forces as functions of the speed ธ:

$$
\begin{aligned}
& A_{3}=\dot{A}_{3} \\
& B_{38}=B_{3} \\
& A_{35}=A_{5}-\frac{0}{2} B P_{3} \\
& B_{35}=B_{35}+\mathrm{DA}_{3} \\
& A_{s 3}=A_{s}+\underbrace{}_{2 B ? s} \\
& B_{53}=B_{35}-\mathrm{uAf}_{3} \\
& A_{55}=A q_{5}+\frac{U^{2}}{\omega^{2}} A l_{3} \\
& B_{55}=B \xi_{5}+{\frac{U^{2}}{2}}_{B q_{3}}
\end{aligned}
$$

The superscript ${ }^{\circ}$ denotes quantities at zero speed. The $A, B^{\circ}$ were found using the M.I.T. Seakeeping prograi [2].

$$
\begin{aligned}
& F_{3}=p \eta^{S}\left(f_{3}+h_{3}\right) d \xi \\
& F_{5}=-p \eta^{S}\left[\xi\left(E_{3}+h_{3}\right)+\frac{\sigma}{1 w} h_{3}\right] d \xi
\end{aligned}
$$

where $f_{f}$ is the sectional Froude-Kryloff force and $h_{j} j$ the sectional diffraction force.

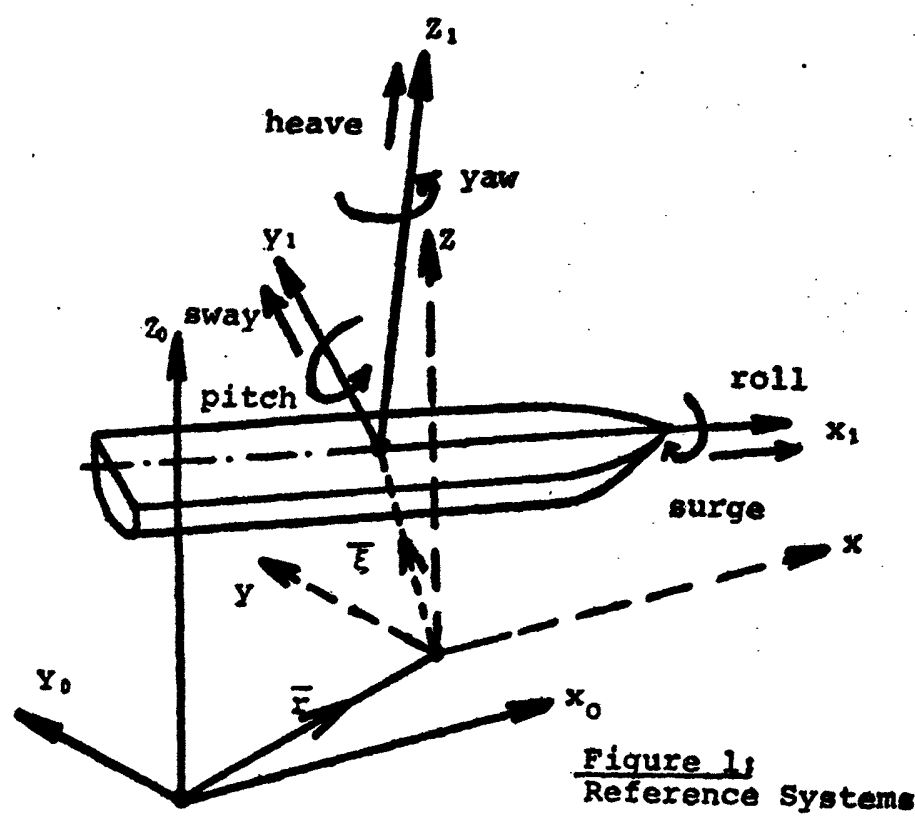



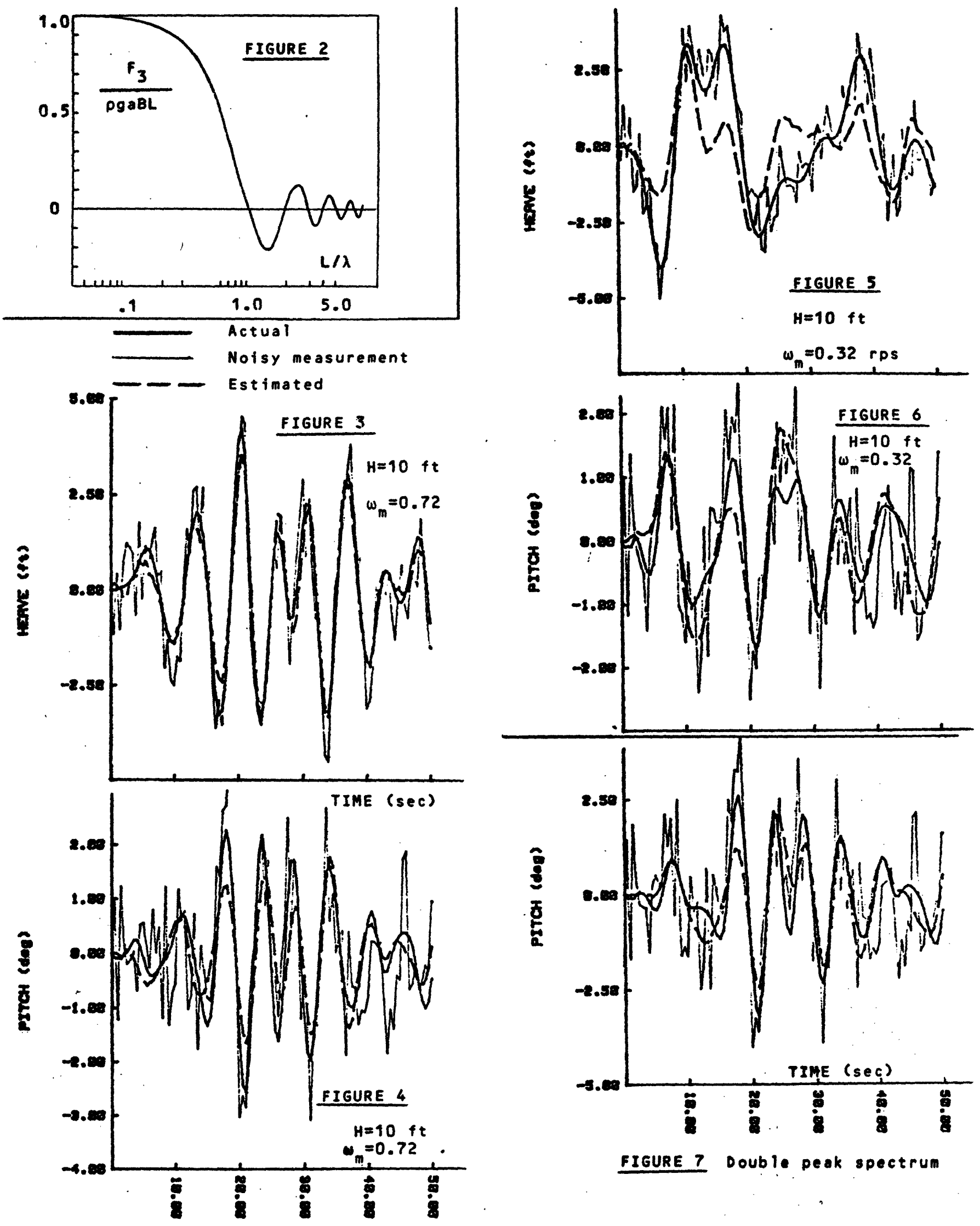